\title{
Pomelo peel oil alleviates cerebral NLRP3 inflammasome activation in a cardiopulmonary resuscitation rat model
}

\author{
XIN-SEN ZOU ${ }^{1 *}$, LU XIE $^{2 *}$, WEN-YAN WANG ${ }^{1}$, GAO-YANG ZHAO $^{1}$, XIN-YUE TIAN $^{1}$ and MENG-HUA CHEN ${ }^{1}$ \\ ${ }^{1}$ Intensive Care Unit, The Second Affiliated Hospital of Guangxi Medical University; \\ ${ }^{2}$ Department of Physiology, Guangxi Medical University, Nanning, Guangxi 530000, P.R. China
}

Received August 14, 2020; Accepted December 11, 2020

DOI: $10.3892 /$ etm.2021.9664

\begin{abstract}
TheNLR family pyrindomain-containing 3(NLRP3) inflammasome, which is composed of NLRP3, apoptosis-associated speck-like protein containing a CARD (ASC) and pro-caspase-1 protein complexes, is activated by the reactive oxygen species (ROS) that are associated with ischemia-reperfusion (I/R) and are involved in brain damage. Pomelo peel oil (PPO) exhibits antioxidant activity. However, it is unclear whether PPO is able to attenuate NLRP3 inflammasome-induced inflammation and pyroptosis. Healthy male Sprague-Dawley rats were subjected to $7 \mathrm{~min}$ of cardiac arrest via trans-esophageal electrical stimulation, followed by cardiopulmonary resuscitation (CPR). The rats were then treated with PPO prior to reperfusion for $24 \mathrm{~h}$. Hematoxylin and eosin staining was used to evaluate brain tissue and cell damage. In the brain tissues, reactive oxygen species (ROS) were assayed, immunofluorescence was used to analyze the expression of NLRP3 and western blotting was performed to determine the expression levels of neuroenolase (NSE), NF- $\kappa \mathrm{B}$, interleukin-1 $\beta$ (IL-1 $\beta$ ), gasdermin D (GSDMD) and the NLRP3 inflammasome. Treatment of the rats with PPO significantly decreased the pathological damage of the brain tissue and reduced the expression of NSE, production of ROS and secretion of NF- $\mathrm{B}$, NLRP3, IL-1 $\beta$ and GSDMD. In conclusion, these results demonstrate the ability of PPO
\end{abstract}

Correspondence to: Professor Meng-Hua Chen, Intensive Care Unit, The Second Affiliated Hospital of Guangxi Medical University, 166 Eastern University Road, Nanning, Guangxi 530000, P.R. China

E-mail:cmhnn@sina.com

${ }^{*}$ Contributed equally

Abbreviations: ROS, reactive oxygen species; I/R, ischemia-reperfusion; PPO, pomelo peel oil; CA, cardiac arrest; CPR, cardiopulmonary resuscitation; H\&E, hematoxylin and eosin; CIRI, cerebral I/R injury; ROSC, restoration of spontaneous circulation; GC-MS, gas chromatography-mass spectrometry

Key words: ischemia-reperfusion injury, NLRP3 inflammasome, inflammation, pyroptosis, cardiopulmonary resuscitation to protect the brain against I/R injury in rats after CPR by a mechanism involving inhibition of the inflammation and pyroptosis mediated by NLRP3 inflammasome activation.

\section{Introduction}

Cardiac arrest (CA) and subsequent cardiopulmonary resuscitation (CPR) induces systemic organ tissue ischemia/reperfusion (I/R) injury, particularly cerebral I/R injury (CIRI), which directly affects the prognosis and quality of life of patients. Numerous pathophysiological mechanisms of I/R injury, including oxidative stress, amino acid toxicity, energy metabolism, calcium overload, apoptosis and autophagy, have been targeted by interventions for brain protection (1-3). In addition, the pathological effects of NLR family pyrin domain-containing 3 (NLRP3) inflammasome-induced inflammation and pyroptosis exacerbate CIRI (4). During I/R, excessive quantities of oxygen free radicals attack tissues or cells and induce damage-associated molecular patterns that are recognized by specific pattern recognition receptors, thereby resulting in caspase- 1 precursor (pro-caspase-1) activation and cell death; this is the classical pathway underlying caspase-1-dependent pyroptosis (5). NLRP3 accompanied by pyroptosis is widely observed in neurons, microglia and astrocytes and is associated with neurological diseases (6). When damage-associated molecular signaling occurs, NLRP3 interacts with apoptosis-associated speck-like protein containing a CARD (ASC) and recruits pro-caspase- 1 . Subsequently, pro-caspase-1 is cleaved to form mature caspase-1p20, which cleaves pro-interleukin (IL)-1 $\beta$ and gasdermin D (GSDMD) to form mature IL-1 $\beta$ p17 and the GSDMD-N domain (GSDMD-N). The GSDMD-N protein has been demonstrated to execute pyroptosis (7). The GSDMD-N domain targets the plasma membrane and forms a pore of diameter 10-14 $\mathrm{nm}$ that allows mature IL-1 $\beta \mathrm{p} 17$ to leak out and ions and water to enter, which causes inflammation and cell pyroptosis (8-11). Therefore, the inhibition of NLRP3 inflammasome activation by antioxidants may be clinically useful for the treatment of I/R injury.

A previous study has demonstrated that, in most organs, including the brain, heart, liver, lungs and intestines, activation of the NLRP3 inflammasome aggravates CIRI (12). Although NLRP3-mediated CIRI has been reported in the middle 
cerebral artery occlusion (MCAO) model (1), it remains unclear whether the global CIRI following CA/CPR, which causes more extensive critical brain damage than MCAO, is associated with the NLRP3 inflammasome and pyroptosis.

Pomelo (Citrus maxima), which is a citrus fruit belonging to the genus Rutaceae, has high nutritional value (13). Traditional Chinese medicine considers pomelo to be beneficial for the prophylaxis and treatment of nervous system disorders, peroxidation damage, cardiovascular disease, bruising, hyperlipidemia, wounds, acne, osteoarthritis and fatigue (14-18). Pomelo peel contains numerous beneficial chemicals, including flavonoids, coumarins, essential oils and limonoids. The essential oils of pomelo peel, extracted using a cold-press method, were found to contain $94.15 \%$ terpenes, comprising $55.92 \%$ limonene, $31.17 \% \beta$-myrcene, $3.16 \% \beta$-pinene, $1.42 \%$ ocimene and $1.24 \% \beta$-copaene, when analyzed using gas chromatography-mass spectrometry (GC-MS) (19). Pomelo peel oils (PPOs) have been demonstrated to exhibit strong antioxidant, anti-inflammatory, antiviral and antibacterial activities (20-22). Therefore, we hypothesize that PPO may inhibit the NLRP3 inflammasome-associated inflammation and pyroptosis induced by CIRI. In the present study, the chemical composition of PPO was analyzed. In addition, the effects of PPO on reactive oxygen species (ROS) and the NLRP3 inflammasome, GSDMD, IL- $1 \beta$ and NF- $\kappa B$ expression were examined in a rat model of $\mathrm{CA} / \mathrm{CPR}$ in order to elucidate the potential neuroprotective effect of PPO.

\section{Materials and methods}

PPO extract. Fresh and mature pomelo peels [C. maxima (Burm.) Merr. cv. Shatian Yu, identified by the Institute of Botany, Guangxi Zhuang Autonomous Region, Chinese Academy of Science; specimen ID: (W.Y.Wang:201909001)] were obtained from the village of Shatian in Rongxia, Guangxi, China. The steam distillation method was used to extract the PPO (23). The outer layer of the pomelo peel was washed and cut into $\sim 5 \times 5-\mathrm{mm}$ pieces. The pieces of peel were combined with distilled water (1:2) and put in a distiller, where they were boiled for $1 \mathrm{~h}$. The PPO was separated from the water using a cooling condenser and stored at $-4^{\circ} \mathrm{C}$.

$G C$-MS analysis. The compounds in the PPO were qualitatively analyzed using GC-MS (TRACE ${ }^{\mathrm{TM}}$ 1300; Thermo Fisher Scientific, Inc.). Helium was injected as a carrier gas at a flow rate of $1 \mathrm{ml} / \mathrm{min}$. The temperature was increased from 45 to $250^{\circ} \mathrm{C}$ according to the procedure. The heating program is maintained at $45^{\circ} \mathrm{C}$ for $1 \mathrm{~min}$, then at $10^{\circ} \mathrm{C} / \mathrm{min}$ to $165^{\circ} \mathrm{C}$ for $2 \mathrm{~min}$, then at $1.5^{\circ} \mathrm{C} / \mathrm{min}$ to $180^{\circ} \mathrm{C}$ for $2 \mathrm{~min}$ and finally at $10^{\circ} \mathrm{C} / \mathrm{min}$ to $250^{\circ} \mathrm{C}$ for $2 \mathrm{~min}$. The temperatures of the injector and detector were set at $250^{\circ} \mathrm{C}$. Mass spectra were scanned from $\mathrm{m} / \mathrm{z}$ 41-400 amu. The electron impact ionization energy was $70 \mathrm{eV}$. Identification of the detected compounds was performed by comparing the mass spectra with published data. Samples were identified using the Retention Time Locked database (NIST MS Search 2.3; http://www.Inchi-trust,org/download/105/licence.pdf) with Deconvolution Reporting Software (MSD ChemStation F. 01.03.2357; 1989-2015; Agilent Technologies, Inc.).
CA/CPR animal model. In total, 60 male Sprague-Dawley (SD) rats (body weight, 220-250 g; age 7-8 weeks) were provided by the Experimental Animal Center of Guangxi Medical University (License number SYXK Gui 2014-0003). All animals were handled in accordance with the Guide for the Care and Use of Laboratory Animals of the National Institutes of Health (24). The study was approved by the Animal Care and Use Committee of Guangxi Medical University. The rats were raised at $22 \pm 2^{\circ} \mathrm{C}$, humidity $50-60 \%, 12$-h light/dark cycle, were free to eat and drink and fasted $12 \mathrm{~h}$ before the operation. Prior to modeling, the rats were anesthetized with an intraperitoneal injection of $2 \%$ pentobarbital sodium $(30 \mathrm{mg} / \mathrm{kg})$. A PE50 tube was inserted into the left femoral artery and connected to a physiological recorder (BL-420E; Chengdu TME Technology Co., Ltd.) via a pressure converter to monitor the blood pressure. Another PE50 tube was inserted into the femoral vein for the injection of drugs.

The CA model was established according to the method reported by Chen et al (25). A temporary pacemaker electrode (Chengdu TME Technology Co., Ltd.) was inserted into the esophagus of the rat at a depth of $\sim 7 \mathrm{~cm}$. Ventricular fibrillation was induced by the application of $12 \mathrm{~V}$ of direct current for $1 \mathrm{~min}$. The criteria used to define CA were as follows: ECG exhibiting ventricular fibrillation and a mean arterial pressure of $<20 \mathrm{mmHg}$. Following $7 \mathrm{~min}$ of CA, CPR was initiated using a frequency of mechanical chest compression of $180 / \mathrm{min}$, with a depth of $25-30 \%$ of the anterior posterior diameter of the chest. Ventilator-assisted ventilation (DH-150; Zhejiang University Medical Instrument Co., Ltd.; https://med4868. yixie8.com/) was applied immediately through endotracheal intubation after $1 \mathrm{~min}$ of CPR (tidal volume, $6 \mathrm{ml} / \mathrm{kg}$; respiration rate, 70 breaths/min; positive end expiratory pressure, $0 \mathrm{cmH}_{2} \mathrm{O}$ ). In addition, $20 \mathrm{mg} / \mathrm{kg}$ epinephrine was administered through the PE50 tube in the femoral vein. The restoration of spontaneous circulation (ROSC) standard was as follows: Supraventricular rhythm (sinus, atrial and borderline heart rhythm) accompanied by a mean arterial pressure of $>60 \mathrm{mmHg}$ lasting for $>1 \mathrm{~min}$. Surviving rats were randomly divided into five groups: NS group $(n=8 ; 0.9 \%$ physiological saline $200 \mu \mathrm{l}$ ), glycerin group (Gly group; $\mathrm{n}=8 ; 10 \%$ glycerin $200 \mu \mathrm{l}$ ), PPO-low group (PPO-L group; $\mathrm{n}=8 ; 10 \mathrm{mg} / \mathrm{kg}$ PPO), PPO-medium group (PPO-M group; $\mathrm{n}=8 ; 20 \mathrm{mg} / \mathrm{kg} \mathrm{PPO}$ ) and PPO-high group (PPO-H; $\mathrm{n}=8 ; 40 \mathrm{mg} / \mathrm{kg}$ PPO). The mass of $1 \mathrm{ml}$ PPO, weighed using an electronic balance (BSA223S; Sartorius AG), was $800 \mathrm{mg}$. In each group treated with PPO, the PPO was combined with $10 \%$ glycerol to a total volume of $200 \mu \mathrm{l}$. Following $1 \mathrm{~min}$ of ROSC, the rats were intravenously injected with the respective treatment. In addition, 8 rats were randomly selected as the sham group, which underwent exposure of the left femoral artery and femoral vein followed by vascular ligation without CA/CPR. All procedures were performed by two skilled operators.

Preparation of brain tissues. All rats were anesthetized using pentobarbital $(30 \mathrm{mg} / \mathrm{kg})$ by intraperitoneal injection $24 \mathrm{~h}$ post reperfusion. Three rats in each group were perfused with $4 \%$ paraformaldehyde for hematoxylin and eosin (H\&E) staining and immunofluorescence experiments. Cerebral cortices were immediately harvested from the remaining 5 rats in each group and stored at $-80^{\circ} \mathrm{C}$ for subsequent western blot analysis. 
ROS assay. Fresh cerebral cortex was homogenized with phosphate buffer in a weight $(\mathrm{g})$ :volume $(\mathrm{ml})$ ratio of 1:20. Following centrifugation at $1,000 \mathrm{x}$ g for $10 \mathrm{~min}$, the supernatant was used for the measurement of ROS and protein concentrations. Thereafter, $190 \mu 1$ supernatant was mixed with $10 \mu 1$ DCFH-DA from an ROS assay kit (WLA070; Wanleibio Co., Ltd.) in a 96-well plate and incubated at $37^{\circ} \mathrm{C}$ in the dark for $30 \mathrm{~min}$. ROS were detected via fluorescence with an excitation wavelength of $500 \mathrm{~nm}$ and emission wavelength of $525 \mathrm{~nm}$. The protein concentration was determined using the BCA method (P0010; Beyotime Institute of Biotechnology) and the results were expressed as fluorescence intensity/mg protein.

Histological assessment. The rat brains fixed with $4 \%$ paraformaldehyde were embedded in paraffin and cut into $3-\mu \mathrm{m}$ coronal sections. These sections were then subjected to H\&E staining. At room temperature, the paraffin sections were dewaxed using xylene followed by a descending ethanol gradient, rehydrated, stained with hematoxylin for $5 \mathrm{~min}$ at $25^{\circ} \mathrm{C}$, reacted with hydrochloric acid ethanol for $5 \mathrm{sec}$ and stained with eosin for $1 \mathrm{~min}$ at $25^{\circ} \mathrm{C}$ before being finally dehydrated. The slices were observed under a light microscope (Olympus, Japan) at a magnification of x400.

Immunofluorescence assessment. The dry 3- $\mu \mathrm{m}$ paraffin slices were subjected to antigen retrieval by heating in a microwave oven at $65^{\circ} \mathrm{C}$ with pH 8.0 EDTA antigen repair buffer (Beijing Solarbio Science \& Technology Co., Ltd.), then incubated in $0.01 \mathrm{M}$ PBS (pH 7.4) containing 0.3\% Triton X-100 (TBST) for $20 \mathrm{~min}$ at $25^{\circ} \mathrm{C}$ prior to blocking in normal goat serum (OriGene Technologies, Inc.) for $30 \mathrm{~min}$ at $37^{\circ} \mathrm{C}$ in $\mathrm{BSA}$ (OriGene Technologies, Inc.). The slices were incubated with anti-NLRP3 primary antibodies (cat. no. w103379; 1:100; Wanleibio Co., Ltd.) overnight at $4{ }^{\circ} \mathrm{C}$, followed by horseradish peroxidase (HRP)-labeled goat anti-rabbit secondary antibodies (cat. nos. GB23303; 1:500; Wuhan Servicebio Technology Co., Ltd.) for $50 \mathrm{~min}$ at $25^{\circ} \mathrm{C}$. FITC-Tyramide (cat. no. G1225-1; 1:1,000; Wuhan Servicebio Technology Co., Ltd.) was added for $10 \mathrm{~min}$ at $25^{\circ} \mathrm{C}$. The sections were then placed into citric acid ( $\mathrm{pH}$ 6.0) antigen repair solution (Wuhan Servicebio Technology Co., Ltd.) and heated in a microwave oven at $65^{\circ} \mathrm{C}$ for $5 \mathrm{~min}$. Anti-allograft inflammatory factor 1 (IBA-1) primary antibodies (cat. no. ab153696; 1:1,000; Abcam) were added to the sections, which were incubated overnight at $4^{\circ} \mathrm{C}$. A Cy3-conjugated fluorescent secondary antibody (cat. no. G1225-2; 1:1,000; Wuhan Servicebio Technology Co., Ltd.) was then added for $50 \mathrm{~min}$ at $25^{\circ} \mathrm{C}$, followed by an autofluorescence quenchant (cat. no. G1221; Wuhan Servicebio Technology Co., Ltd.) for $5 \mathrm{~min}$. Immunoreactivity was visualized by the fluorescence of the dye to which the secondary antibodies were conjugated. DAPI dye (cat. no. G1012; Wuhan Servicebio Technology Co., Ltd.) was added and the slices were incubated for $10 \mathrm{~min}$ at $25^{\circ} \mathrm{C}$ in the absence of light. The slices were sealed with an anti-fluorescence quenching sealant (cat. no. G1401; Wuhan Servicebio Technology Co., Ltd.), and images were captured using a fluorescence microscope (Olympus Corporation) with an excitation wavelength of 465-495 nm for NLRP3 (green), 510-560 nm for IBA-1 (red) and 330-380 nm (blue) for DAPI staining. Three magnification fields $(\mathrm{x} 400)$ in the slices were randomly selected. The NLRP3-positive area (\%), Iba-1 positive area (\%) and number of microglia with co-localized NLRP3 and IBA-1 expression were determined using ImageJ 6.0 software (National Institutes of Health).

Western blot analysis of neuroenolase (NSE), NF- $\kappa$ Bp105/p50, NLRP3, ASC, caspase-1, IL-1 $\beta$ and GSDMD. Brain tissue samples (50 mg) were lysed by RIPA Lysis Buffer (Wuhan Servicebio Technology Co, Ltd.) and centrifuged at $12,000 \mathrm{x}$ g for $15 \mathrm{~min}$ at $4^{\circ} \mathrm{C}$ and the supernatant was collected. The protein concentration was determined using a bicinchoninic acid protein assay kit (Beyotime Institute of Biotechnology). Protein samples (40 $\mu \mathrm{g} / \mathrm{lane})$ were separated via SDS-PAGE (12 or $8 \%$ separation gel) and then transferred to a PVDF membrane (Merck KGaA). The membranes were incubated overnight at $4{ }^{\circ} \mathrm{C}$ with the following primary antibodies: NSE (cat. no. ab53025; 1:1,000; Abcam), NF-кBp105/p50 (cat. no. ab32360; 1:1,000; Abcam), NFкBp105/p50 (phospho S337; cat. no. ab194729; 1:1,000; Abcam), NLRP3 (cat. no. wl03379; 1:1,000; Wanleibio Co., Ltd.), ASC (cat. no. w102462; 1:500; Wanleibio Co., Ltd.), caspase-1 (cat. no. w103325; 1:500; Wanleibio Co., Ltd.), IL-1 $\beta$ (cat. no. ab9787; 1:1,000; Abcam) and GSDMD (cat. no. ab219800; 1:1,000; Abcam) and GAPDH (cat. no. 5174, 1:1,000; Cell Signaling Technologies, Inc.). After washing with TBST $(0.1 \%$ tween), the membranes were incubated with HRP-conjugated goat anti-rabbit secondary antibodies (cat. no. sc-2004; 1:10,000; Santa Cruz Biotechnology, Inc.) for $1 \mathrm{~h}$ at $25^{\circ} \mathrm{C}$. Proteins were detected using the Tanon ${ }^{\mathrm{TM}}$ High-sig ECL Western Blotting Substrate (Guangzhou Yuwei Biotechnology Instrument Co., Ltd.). Image J 6.0 software was used to analyze the intensities of the bands.

Statistical analysis. All data are expressed as mean \pm standard error of the mean (SEM). Statistical analyses were performed using GraphPad Prism 7 (GraphPad Software, Inc.). The Shapiro-Wilk test was used to validate assumptions of normality. One-way ANOVA followed by a Tukey's multiple comparison test was employed to analyze differences among groups. The Kruskal-Wallis test was used to evaluate non-normally distributed data, with Dunn's test for intergroup comparisons. $\mathrm{P}<0.05$ was considered to indicate a statistically significant differences.

\section{Results}

Chemical composition of PPO. The extraction yield of PPO was $0.2 \%$. The PPO was obtained as a faint yellow, transparent liquid. The total ion chromatogram obtained from the GC-MS analysis of the PPO is shown in Fig. 1. A total of 17 compounds were detected (Table I). The most abundant compound in the PPO was limonene at $88.683 \%$, followed by nootkatone $(5.732 \%)$ and myrcene (1.027\%; Table I).

Antioxidant activities of PPO. The antioxidant activities of PPO in the cerebral cortex tissues of the CIRI model rats are shown in Fig. 2B. In comparison with the ROS content in the sham group, the levels of ROS were significantly increased in the NS, Gly and PPO-L groups. However, in the PPO-H group, the PPO treatment significantly decreased the levels of ROS compared with those in the NS and Gly groups. 


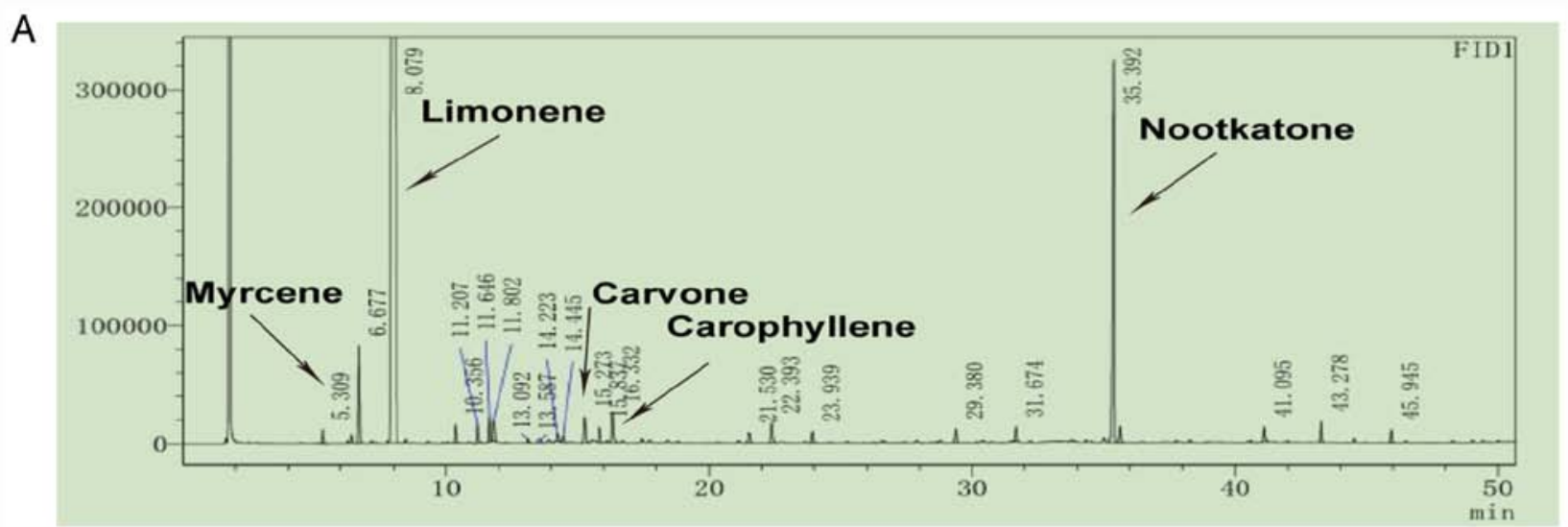

GC spectrum of PPO
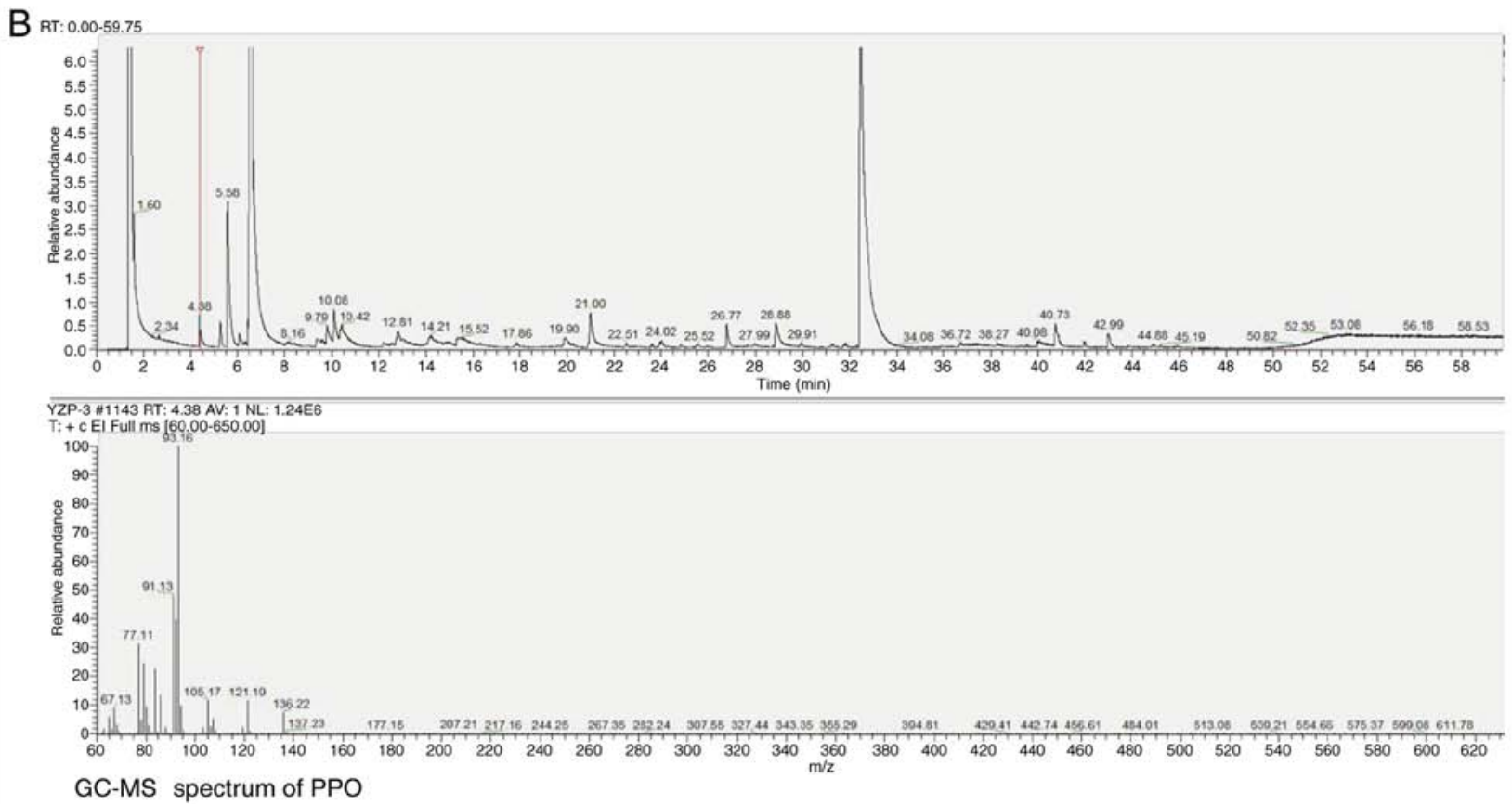

Figure 1. PPO constituents detected by GC-MS and identified by comparison with purified compound standards. (A) GC spectrum of PPO. The main components are indicated by arrows. (B) GC-MS spectrum of PPO. PPO, pomelo peel oil; GC, gas chromatography; MS, mass spectrometry.

PPO ameliorates cerebral cell morphological changes and reduces the expression of NSE. To investigate the effects of PPO on CIRI, cerebral cell morphology was evaluated with H\&E staining (Fig. 2A). In the sham group, the cell structures were complete and the tissue structure was tightly packed, with uniform staining and clearly visible nuclei. By contrast, the cerebral tissues from the NS and Gly groups exhibited fewer intact cells; cells were disordered, with intense staining and vacuoles clearly evident. The tissues treated with PPO exhibited improved cell morphology compared with those in the NS and Gly groups. NSE has been widely studied as a brain injury marker $(21,26)$. Therefore, the expression of NSE was analyzed by western blotting (Fig. 2C). Compared with that in the sham group, the expression of NSE was significantly increased in the NS, Gly, PPO-L and PPO-M groups (Fig. 2D). However, the expression of NSE in the PPO-H group was significantly lower than that in the NS, Gly and PPO-L groups (Fig. 2D). These results indicate that is able to PPO ameliorate cerebral injury in $\mathrm{CA} / \mathrm{CPR}$ model rats.
$P P O$ inhibits the expression of $N F-\kappa B$. When NF- $\kappa \mathrm{Bp} 105$ is lysed, it forms NF- $\kappa \mathrm{Bp} 50$ and thereby serves pro-inflammatory functions (27). No significant differences in the expression of NF- $\kappa$ Bp105 were detected among the groups (Fig. 2E and F). However, in comparison with the sham group, the NS and Gly groups exhibited significant increases in

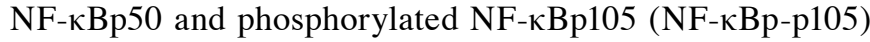
levels (Fig. 2G-I). Following treatment with PPO, the levels

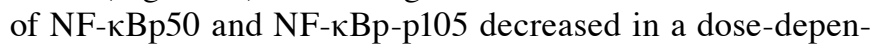
dent manner, and the level of NF- $\mathrm{kBp}-\mathrm{p} 105$ was significantly decreased in the PPO-H group compared with the NS and

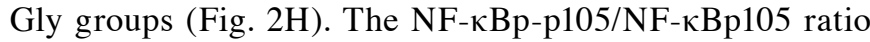
also exhibited a significant reduction in the PPO-H group compared with the NS and Gly groups after PPO treatment (Fig. 2I). These results suggest that PPO partially inhibits NF- $\mathrm{kB}$-mediated inflammation.

PPO decreases the expression of NLRP3 in the cerebral cortex. To determine whether PPO is able to inhibit NLRP3 
Table I. Chemical components of pomelo peel oil.

\begin{tabular}{|c|c|c|c|c|}
\hline No. & Compound & Molecular formula & Retention time (min) & Peak area $(\%)$ \\
\hline 1 & $\alpha$-pinene & $\mathrm{C}_{10} \mathrm{H}_{16}$ & 5.309 & 0.127 \\
\hline 2 & Myrcene & $\mathrm{C}_{10} \mathrm{H}_{16}$ & 6.677 & 1.027 \\
\hline 3 & Limonene & $\mathrm{C}_{10} \mathrm{H}_{16}$ & 8.079 & 88.683 \\
\hline 4 & $\gamma$-terpinene & $\mathrm{C}_{10} \mathrm{H}_{16}$ & 10.356 & 0.250 \\
\hline 5 & Limonene oxide & $\mathrm{C}_{10} \mathrm{H}_{16} \mathrm{O}$ & 11.207 & 0.270 \\
\hline 6 & $\alpha$-terpineol & $\mathrm{C}_{10} \mathrm{H}_{18} \mathrm{O}$ & 14.223 & 0.149 \\
\hline 7 & Carvone & $\mathrm{C}_{10} \mathrm{H}_{14} \mathrm{O}$ & 15.273 & 0.403 \\
\hline 8 & Geranyl acetate & $\mathrm{C}_{12} \mathrm{H}_{20} \mathrm{O}_{2}$ & 15.837 & 0.234 \\
\hline 9 & Carophyllene & $\mathrm{C}_{15} \mathrm{H}_{24}$ & 16.332 & 0.505 \\
\hline 10 & $\alpha$-caryophyllene & $\mathrm{C}_{15} \mathrm{H}_{24}$ & 21.530 & 0.193 \\
\hline 11 & $\alpha$-gurjunene & $\mathrm{C}_{15} \mathrm{H}_{24}$ & 22.393 & 0.351 \\
\hline 12 & 8-cedren-13-ol & $\mathrm{C}_{15} \mathrm{H}_{24} \mathrm{O}$ & 23.939 & 0.183 \\
\hline 13 & Nerolidol & $\mathrm{C}_{15} \mathrm{H}_{26} \mathrm{O}$ & 29.380 & 0.208 \\
\hline 14 & Globulol & $\mathrm{C}_{15} \mathrm{H}_{26} \mathrm{O}$ & 31.674 & 0.184 \\
\hline 15 & Nootkatone & $\mathrm{C}_{15} \mathrm{H}_{22} \mathrm{O}$ & 35.392 & 5.732 \\
\hline 16 & Osthole & $\mathrm{C}_{15} \mathrm{H}_{16} \mathrm{O}_{3}$ & 41.095 & 0.196 \\
\hline 17 & Eicosane & $\mathrm{C}_{20} \mathrm{H}_{42}$ & 43.278 & 0.197 \\
\hline- & Total & - & - & 98.89 \\
\hline
\end{tabular}

activation in the microglia, the expression and co-expression of NLRP3 and IBA-1 were detected using immunofluorescence double staining. The expression of NLRP3 and IBA-1, and the co-expression of NLRP3 + IBA-1 increased markedly in the microglia following CR/CPR (Fig. 3A). The expression of NLRP3, IBA-1 and NLRP3 + IBA-1 was significantly increased in the NS and Gly groups compared with the sham group. After treatment with PPO, the expression levels of NLRP3, IBA-1 and NLRP3 + IBA-1 in the PPO-H group were significantly lower than those in the NS group, indicating that activation of microglia was inhibited (Fig. 3B). The results indicate that PPO decreased the expression of NLRP3 and activation of the microglia.

PPO downregulates the expression of pyroptosis-associated proteins NLRP3, ASC, caspase-1, IL-1 $\beta$ and GSDMD. To investigate the effects of PPO on the inflammatory response and pyroptosis-associated proteins in the CA/CPR model rats, the levels of NLRP3, ASC, caspase-1, IL-1 $\beta$ and GSDMD were analyzed using western blotting (Fig. 4). Pro-caspase-1, pro-IL-1 $\beta$ and GSDMD were activated to form caspase-1p20, IL-1ßp17 and GSDMD-N, respectively. The expression levels of NLRP3, ASC, caspase-1p20, IL-1ßp17 and GSDMD-N in the NS group were significantly higher than those in the sham group. Following treatment with PPO, the levels of these proteins were significantly lower than those in the NS group. These data indicate that PPO reduced the pyroptosis-associated protein cascade.

\section{Discussion}

In the present study, PPO was obtained from fresh and mature Shatian pomelo peels. The main ingredient of PPO was found to be limonene $(88.683 \%)$, followed by nootkatone $(5.732 \%)$ and myrcene (1.027\%). The results of the in vivo experiments revealed that PPO reduced the production of ROS induced by global CIRI in rats following CA/CPR and ameliorated the pathological injury of the cerebral cortex. The effects of PPO on the NLRP3 inflammasome response and pyroptosis were also demonstrated in this model. Furthermore, PPO downregulated pyroptosis-associated protein levels.

The proportions of limonene and other components detected in the present study differ from those in other studies $(19,28)$; this may be due to different extraction methods, growth environments, isolation conditions and provenance. However, consistent with other studies $(19,29)$, limonene was the main component of the PPO extracted via distillation in the present study. PPO has been reported to contain $94.15 \%$ terpenes (19), with structures that include phenolic hydroxyl groups and unsaturated double bonds, thereby providing electrons that are able to reduce free radicals to form less active substances and chelate metal ions to prevent the generation of free radicals (30). PPO has also been shown to exert an antioxidant effect against superoxide anion radical formation (19). Previous studies have demonstrated that the overproduction of ROS is associated with CIRI $(31,32)$, which is consistent with the results of the present study. Furthermore, the results indicate that PPO inhibited the production of ROS associated with global CIRI. It has been reported that limonene has antioxidant activities (33). Therefore, we hypothesize that the antioxidant activity of PPO is associated with the limonene it contains.

The generation of excessive ROS leads to cellular damage and triggers the activation of microglia and immune pathways (34). The present study demonstrated that CIRI upregulated the expression of NLRP3 in the microglia, and treatment with PPO reduced CIRI-induced activation of the microglia and the expression of NLRP3. The microglia are 

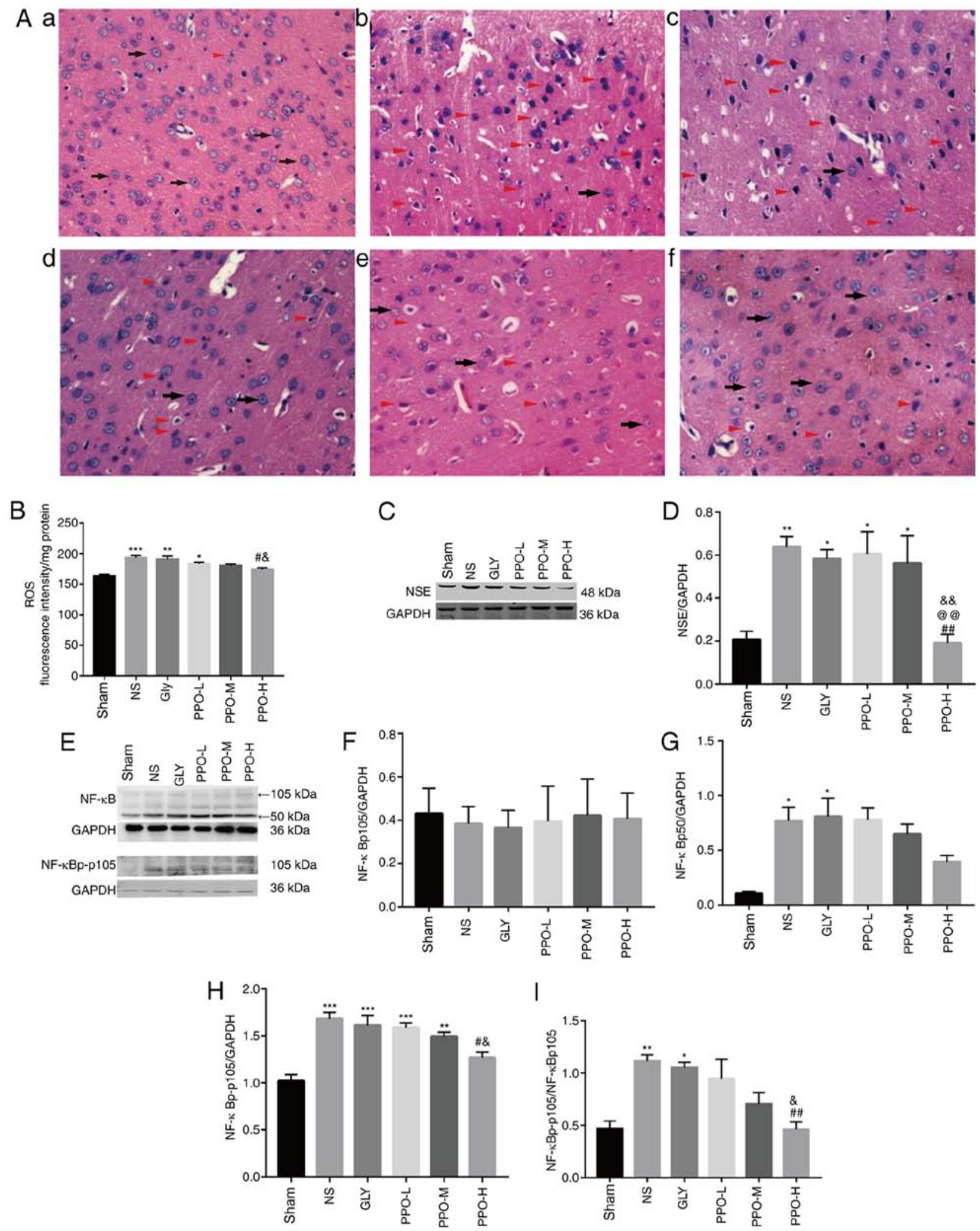

Figure 2. PPO improves cell morphology, reduces ROS levels and attenuates the expression of NSE and NF- $\mathrm{kB}$ in a cardiopulmonary resuscitation rat model. (A) Hematoxylin and eosin staining of brain tissue from rats in the (A-a) sham, (A-b) NS, (A-c) Gly, (A-d) PPO-L, (A-e) PPO-M and (A-f) PPO-H groups. A large number of cerebral cortex cells with normal morphology were observed in the sham group (indicated by black arrows). However, numerous abnormal cerebral cortex cells exhibiting nuclear pyknosis, intense staining, vacuolation, swelling and necrosis (indicated by red triangles) were visible in the NS and Gly groups. Cell morphology was ameliorated in the PPO groups. Magnification, x400. (B) Antioxidant activity of PPO determined by an ROS assay. Representative western blots of NSE (C) and (D) quantified results. (E) Representative western blots of NF- $\mathrm{kB}$ and (F-I) quantified results. Band intensities

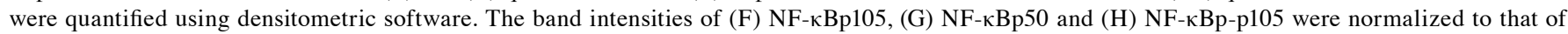
GAPDH, and (I) the NF- $\kappa B p-p 105 / \mathrm{NF}-\kappa B p 105$ ratio was calculated. Data are presented as the mean \pm SEM of two independent experiments ( $\mathrm{n}=3$ ). $\mathrm{P}<0.05$, ${ }^{* *} \mathrm{P}<0.01$ and ${ }^{* * *} \mathrm{P}<0.001$ vs. the sham group; ${ }^{\#} \mathrm{P}<0.05$ and ${ }^{\# \#} \mathrm{P}<0.01$ vs. the NS group; ${ }^{\circledR} \mathrm{P}<0.05$ and ${ }^{\& \&} \mathrm{P}<0.01$ vs. the Gly group; ${ }^{\circledR} \mathrm{e}<0.01$ vs. the $\mathrm{PPO}-\mathrm{L}$ group $(\mathrm{n}=5)$. PPO, pomelo peel oil; ROS, reactive oxygen species; NSE, neuroenolase; NS, physiological saline; Gly, glycerin; PPO-L, $10 \mathrm{mg} / \mathrm{kg}$ PPO; PPO-M, $20 \mathrm{mg} / \mathrm{kg}$ PPO; PPO-H, 40 mg/kg PPO; NF-kBp-p105, phosphorylated NF-kBp105. 
A

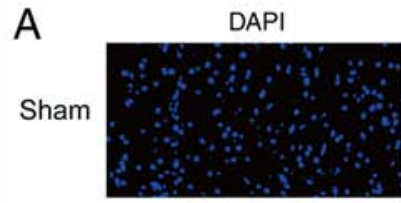

NS

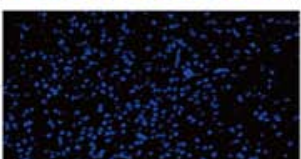

Gly
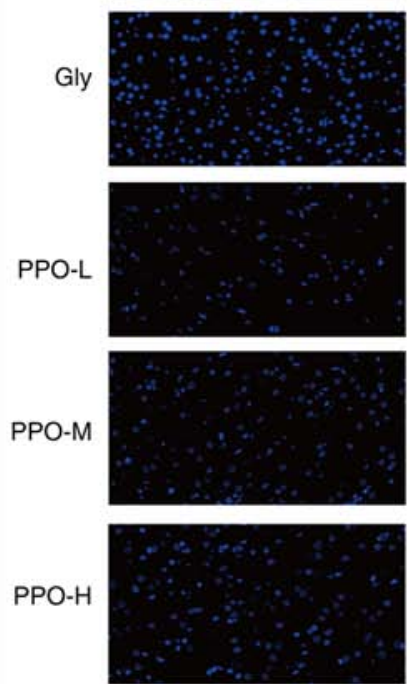

B

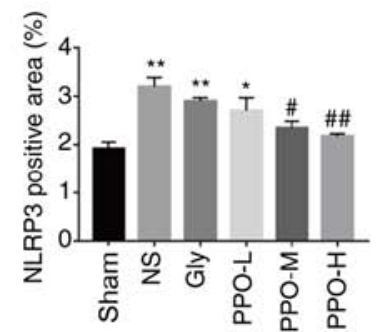

NLRP3
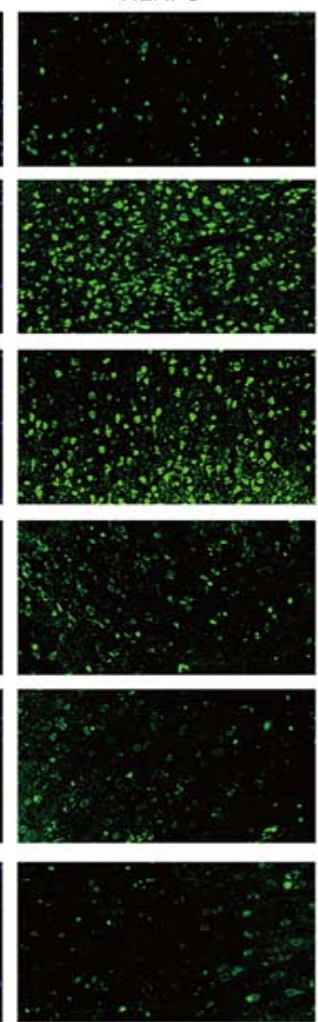

IBA-1
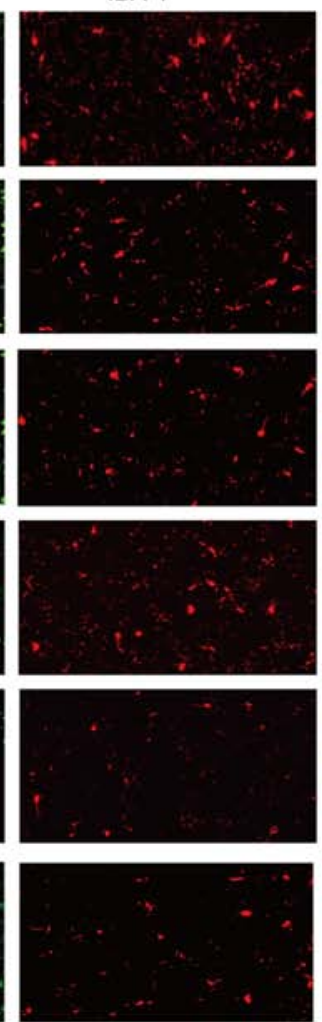

b

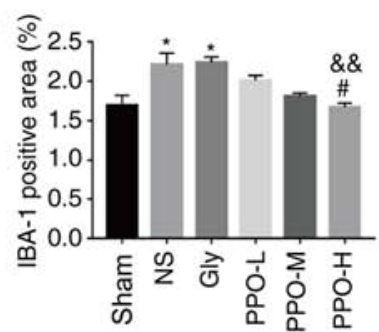

Merge
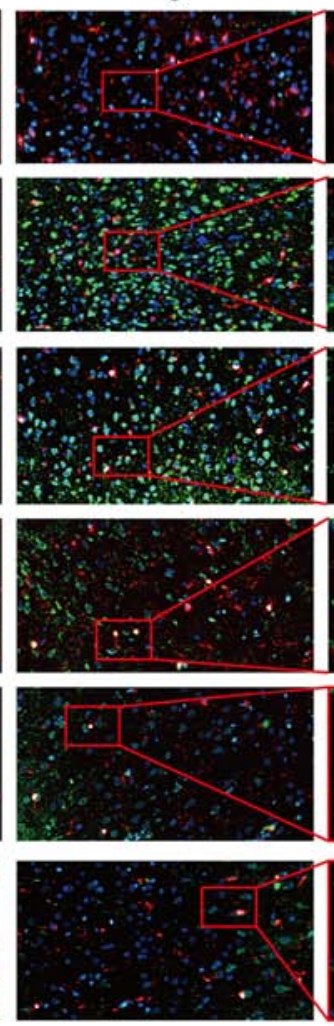

C

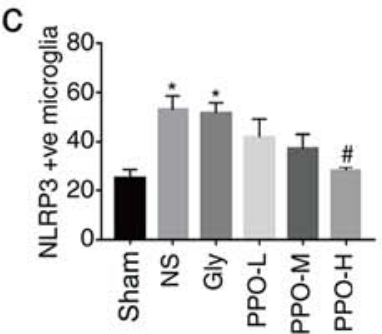

Figure 3. PPO decreases the expression of NLRP3 in the microglia. (A) Immunofluorescence staining of rat brain slices. Green staining indicates NLRP3 protein expression, red staining indicates IBA-1 protein expression and DAPI staining indicates nuclei (blue). Magnification, $\mathrm{x} 400$ and $\mathrm{x} 2,000$. (B) Column charts showing the (B-a) NLRP3-positive area (\%), (B-b) IBA-1-positive area (\%) and (B-c) the amount of NLRP3 expression co-localized with IBA-1-positive microglia in each group quantified using image analysis software. All data are presented as the mean $\pm \mathrm{SEM}\left(\mathrm{n}=3\right.$ ). ${ }^{*} \mathrm{P}<0.05$ and ${ }^{* *} \mathrm{P}<0.01$ vs. the sham group; ${ }^{\#} \mathrm{P}<0.05$ and ${ }^{\# \#} \mathrm{P}<0.01$ vs. the NS group; ${ }^{\&} \mathrm{P}<0.01$ vs. the Gly group. PPO, pomelo peel oil; NLRP3, NLR family pyrin domain-containing 3 ; IBA-1, allograft inflammatory factor 1; Gly, glycerin; PPO-L, $10 \mathrm{mg} / \mathrm{kg}$ PPO; PPO-M, $20 \mathrm{mg} / \mathrm{kg}$ PPO; PPO-H, 40 mg/kg PPO.

the resident immune cells in the brain. Following activation, microglia switch their phenotypes between two polarizations, M1 and M2, which can release large quantities of pro-inflammatory and anti-inflammatory cytokines, respectively. In a previous study, NLRP3 immunoreactivity was detected in the IBA-1-labeled microglia of mice with CIRI following transient MCAO, which induced the overproduction of the M1 microglia-regulated pro-inflammatory cytokine IL-1 $\beta$ (35). Therefore, the phenotypic changes occurring in the microglia following activation require identification.

NLRP3, a specific pattern recognition receptor, initiates cell death processes (pyroptosis) as soon as it is activated by factors induced by pathological damage. It promotes the pyroptosis-associated protein cascade and causes inflammation and pyroptosis (36). The examination of factors in this cascade in the present study revealed that the upregulation of NLRP3, ASC, caspase-1p20, IL-1ßp17 and GSDMD-N induced in rats by CA/CPR was attenuated by PPO treatment, suggesting that it targets NLRP3 inflammasome induced inflammatory responses and pyroptosis, and serves as an intervention against CIRI.

The control of NLRP3 activity can be divided into two processes, namely priming and activation. Priming prepares NLRP3 for subsequent activation (37) and may occur by an ROS-mediated non-transcriptional pathway $(38,39)$. ROS released into the cytoplasm by damaged mitochondria also promote activation of the NLRP3 inflammasome $(40,41)$. Since excess ROS production occurs during CIRI, antioxidants are able block pyroptosis-associated NLRP3 inflammasomes. Therefore, we hypothesize that the antioxidant activity of PPO resulted in the levels of NLRP3 inflammasome being low in the early stages of CIRI.

IL-1 $\beta$ is produced during CIRI; it induces cerebrovascular endothelial cells to express adhesion molecules, which mediate 

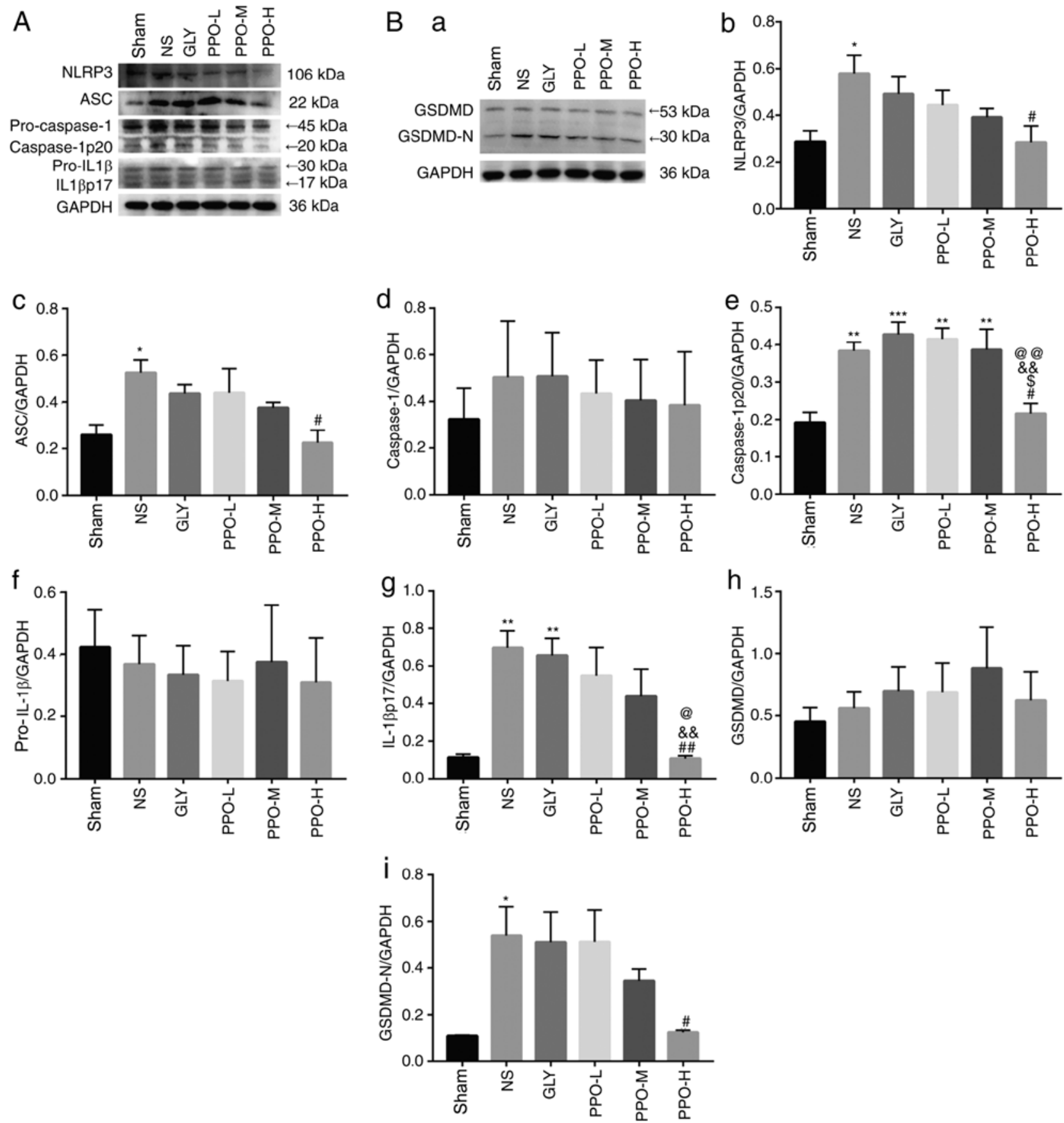

Figure 4. Effect of PPO on NLRP3, ASC, caspase-1, IL-1 $\beta$, and GSDMD in rat brain tissue. Representative western blots of (A) NLRP3, ASC, caspase-1, IL-1 $\beta$ and (B-a) GSDMD. The column charts shows the levels of (B-b) NLRP3, (B-c) ASC, (B-d) caspase-1, (B-e) caspase-1p20, (B-f) pro-IL-1 $\beta$, (B-g) IL-1/p17, (B-h) GSDMD and (B-i) GSDMD-N in the various treatment groups. Band intensity was normalized to GAPDH and quantified using image analysis software. Data are presented as the mean \pm SEM $(n=5) .{ }^{*} \mathrm{P}<0.05,{ }^{* *} \mathrm{P}<0.01$ and ${ }^{* * *} \mathrm{P}<0.001$ vs. the sham group; ${ }^{*} \mathrm{P}<0.05$ and ${ }^{\# \#} \mathrm{P}<0.01$ vs. the NS group; \&\& $\mathrm{P}<0.01$ vs. the Gly group; ${ }^{\circledR} \mathrm{P}<0.05$ and ${ }^{\circledR}{ }^{\circledR} \mathrm{P}<0.01$ vs. the PPO-L group; ${ }^{\mathrm{S}} \mathrm{P}<0.05$ vs. the PPO-M group. PPO, pomelo peel oil; NLRP3, NLR family pyrin domain-containing 3; ASC, apoptosis-associated speck-like protein containing a CARD; IL-1b, interleukin-1b; GSDMD, gasdermin D; GSDMD-N, GSDMD N-domain; Gly, glycerin; PPO-L, 10 mg/kg PPO; PPO-M, 20 mg/kg PPO; PPO-H, 40 mg/kg PPO.

interaction between the endothelial cells and leukocytes, thereby promoting the infiltration of leukocytes into the brain tissues. Leukocyte infiltration activates microglial cells, which causes them to secrete inflammatory factors and aggravates brain injury. In addition, leukocytes adhere and accumulate in microvessels, causing blockages and reducing cerebral blood flow, which also aggravates brain injury. These pathological changes occur at a late stage after CIRI (42-45). We hypothesize that the antioxidant activity of PPO reduces the secretion of IL- $1 \beta$ from cells and thus attenuates of the IL-1 $\beta$-mediated inflammatory response.

An increase in ROS levels can stimulate the phosphorylation of I $\mathrm{B}$ by casein kinase II or tyrosine kinases, and thereby induce $\mathrm{NF}-\mathrm{\kappa B}$ gene transcription (46). NF- $\mathrm{\kappa B}$ mediates the priming of NLRP3 via the transcriptional pathway (39). NF- $\kappa B p 105 / p 50$ is a member of the NF- $\kappa B$ family, and NF- $\kappa B p 50$ is produced from NF- $\kappa B p 105$ by ubiquitin-dependent limited proteolysis (27). The present study found that PPO significantly downregulated 
the phosphorylation of $\mathrm{NF}-\kappa \mathrm{B}$. The phosphorylation of

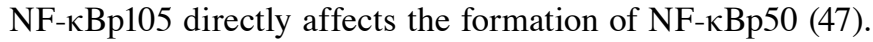
$\mathrm{NF}-\kappa \mathrm{Bp} 50$ has been demonstrated to be involved in pyroptosis as an inflammasome promoter (48). Furthermore, a recent study indicated that the brain damage in MCAO rat models of CIRI may be associated with increased levels of NF- $\kappa$ Bp50 (49). The results of the present study are consistent with this, and showed that the levels of NF- $\kappa$ Bp50 increased in a global CIRI model. PPO downregulated the formation of NF- $\kappa$ Bp50 in a dose-dependent manner; the antipyroptotic properties of PPO may be partly due to its antioxidant activity. Since significant differences were found, the results suggest that the transcriptional pathway of NF- $\mathrm{B}$-mediated NLRP3 activation may be one of the pathway in the present model.

In conclusion, the present study indicated that the NLRP3 inflammasome, which is associated with pyroptosis, is involved in CA/CPR-induced CIRI. Furthermore, PPO treatment downregulated the expression of multiple factors in the NLRP3 inflammasome and ameliorated brain cell death. PPO, as a mixture, may inhibit the inflammation and pyroptosis caused by activation of the NLRP3 inflammasome through its antioxidant capacity. However, further research is required to identify the active components and the underlying mechanism.

\section{Acknowledgements}

Not applicable.

\section{Funding}

The present study was supported by the National Natural Science Foundation of China (grant nos. 81660312 and 81360286).

\section{Availability of data and materials}

All data generated and/or analyzed during this study are included in this published article.

\section{Authors' contributions}

XSZ and LX contributed equally to this work, in terms of developing the concept and study design, acquiring, analyzing and interpreting the data, authenticate the raw data and drafting the manuscript. WYW, GYZ and XYT performed the experiments and analyzed the data. MHC designed and led the study. All authors read and approved the final manuscript.

\section{Ethics approval and consent to participate}

The study was approved by the Animal Care and Use Committee of Guangxi Medical University (Nanning, China).

\section{Patient consent for publication}

Not applicable.

\section{Competing interests}

The authors declare that they have no competing interests.

\section{References}

1. He Q, Li Z, Wang Y, Hou Y, Li L and Zhao J: Resveratrol alleviates cerebral ischemia/reperfusion injury in rats by inhibiting NLRP3 inflammasome activation through Sirt1-dependent autophagy induction. Int Immunopharmacol 50: 208-215, 2017.

2. Chumboatong W, Thummayot S, Govitrapong P, Tocharus C, Jittiwat $\mathrm{J}$ and Tocharus J: Neuroprotection of agomelatine against cerebral ischemia/reperfusion injury through an antiapoptotic pathway in rat. Neurochem Int 102: 114-122, 2017.

3. Buckley KM, Hess DL, Sazonova IY, Periyasamy-Thandavan S, Barrett JR, Kirks R, Grace H, Kondrikova G, Johnson MH, Hess DC, et al: Rapamycin up-regulation of autophagy reduces infarct size and improves outcomes in both permanent MCAL, and embolic MCAO, murine models of stroke. Exp Transl Stroke Med 6: 8, 2014

4. Ito M, Shichita T, Okada M, Komine R, Noguchi Y, Yoshimura A and Morita R: Bruton's tyrosine kinase is essential for NLRP3 inflammasome activation and contributes to ischaemic brain injury. Nat Commun 6: 7360, 2015.

5. Bi F, Ma H, Ji C, Chang C, Liu W and Xie K: Rhein protects against neurological deficits after traumatic brain injury in mice via inhibiting neuronal pyroptosis. Front Pharmacol 11: 564367, 2020.

6. Albornoz E, Woodruff T and Gordon R: Inflammasomes in CNS diseases. Exp Suppl 108: 41-60, 2018.

7. Shi J, Zhao Y, Wang K, Shi X, Wang Y, Huang H, Zhuang Y, Cai T, Wang F and Shao F: Cleavage of GSDMD by inflammatory caspases determines pyroptotic cell death. Nature 526: 660-665, 2015

8. Ding J, Wang K, Liu W, She Y, Sun Q, Shi J, Sun H, Wang DC and Shao F: Pore-forming activity and structural autoinhibition of the gasdermin family. Nature 535: 111-116, 2016.

9. Fink SL and Cookson BT: Caspase-1-dependent pore formation during pyroptosis leads to osmotic lysis of infected host macrophages. Cell Microbiol 8: 1812-1825, 2006.

10. Tsuchiya $\mathrm{K}$ and Hara $\mathrm{H}$ : The inflammasome and its regulation. Crit Rev Immunol 34: 41-80, 2014.

11. Agostini L, Martinon F, Burns K, McDermott MF, Hawkins PN and Tschopp J: NALP3 forms an IL-1beta-processing inflammasome with increased activity in Muckle-Wells autoinflammatory disorder. Immunity 20: 319-325, 2004.

12. Minutoli L, Puzzolo D, Rinaldi M, Irrera N, Marini H, Arcoraci V, Bitto A, Crea G, Pisani A, Squadrito F, et al: ROS-mediated NLRP3 inflammasome activation in brain, heart, kidney, and testis ischemia/reperfusion injury. Oxid Med Cell Longev 2016: $2183026,2016$.

13. Liu Y, Liu A, Ibrahim SA, Yang H and Huang W: Isolation and characterization of microcrystalline cellulose from pomelo peel. Int J Biol Macromo 111: 717-721, 2018.

14. Aumeeruddy-Elalfi Z, Gurib-Fakim A and Mahomoodally MF: Kinetic studies of tyrosinase inhibitory activity of 19 essential oils extracted from endemic and exotic medicinal plants. South Afr J Botany 103: 89-94, 2016.

15. Kim GS, Park HJ, Woo JH, Kim MK, Koh PO, Min W, Ko YG, $\mathrm{Kim} \mathrm{CH}$, Won $\mathrm{CK}$ and Cho JH: Citrus aurantium flavonoids inhibit adipogenesis through the Akt signaling pathway in 3T3-L1 cells. BMC Complement Altern Med 12: 31, 2012.

16. Liu Y, Heying E and Tanumihardjo SA: History, global distribution, and nutritional importance of citrus fruits. Compre Rev Food Sci Food Safety 11: 530-545, 2012.

17. Mulero J, Bernabé J, Cerdá B, García-Viguera C, Moreno DA, Albaladejo MD, Avilés F, Parra S, Abellán J and Zafrilla P: Variations on cardiovascular risk factors in metabolic syndrome after consume of a citrus-based juice. Clin Nutr 31: 372-377, 2012.

18. Hwang SL, Shih PH and Yen GC: Neuroprotective effects of citrus flavonoids. J Agric Food Chem 60: 877-885, 2012.

19. He W, Li X, Peng Y, He X and Pan S: Anti-oxidant and anti-melanogenic properties of essential oil from peel of pomelo cv. Guan Xi. Molecules 24: 242, 2019.

20. Hosni K, Zahed N, Chrif R, Abid I, MedfeiW, Kallel M, Kallel NB and Sebei H: Composition of peel essential oils from four selected Tunisian Citrus species: Evidence for the genotypic influence. Food Chemistry 123: 1098-1104, 2010.

21. Wu F, Jin Y, Xu X and Yang N: Electrofluidic pretreatment for enhancing essential oil extraction from citrus fruit peel waste. J Clean Prod 159: 85-94, 2017.

22. Zhao YL, Yang XW, Wu BF, Shang JH, Liu YP, Zhi-Dai and Luo XD: Anti-inflammatory effect of Pomelo Peel and its Bioactive Coumarins. J Agric Food Chem 67: 8810-8818, 2019. 
23. Fadel H, Marx F, El-Sawy A and El-Ghorab A: Effect of extraction techniques on the chemical composition and antioxidant activity of Eucalyptus camaldulensis var. brevirostris leaf oils. Zeitschrift für Lebensmitteluntersuchung und -Forschung A 208: 212-216, 1999.

24. National Research Council (US): Institute for Laboratory Animal Research. Guide for the Care and Use of Laboratory Animals. National Academies Press (US), Washington, DC, 1996.

25. Chen MH, Liu TW, Xie L, Song FQ, He T, Zeng ZY and Mo SR: A simpler cardiac arrest model in rats. Am J Emerg Med 25 . 623-630, 2007.

26. Abdel-Magied N, Shedid SM and Ahmed AG: Mitigating effect of biotin against irradiation-induced cerebral cortical and hippocampal damage in the rat brain tissue. Environ Sci Pollut Res Int 26: 13441-13452, 2019.

27. Cartwright T, Perkins ND and L Wilson C: NFKB1: A suppressor of inflammation, ageing and cancer. FEBS J 283: 1812-1822, 2016.

28. Ali MY, Rumpa NN, Paul S, Hossen MS, Tanvir EM, Hossan T, Saha M, Alam N, Karim N, Khalil MI and Gan S: Antioxidant potential, subacute toxicity, and beneficiary effects of methanolic extract of pomelo (Citrus grandis L. Osbeck) in long evan rats J Toxicol 2019: 2529569, 2019.

29. Chen GW, Lin YH, Lin CH and Jen HC: Antibacterial activity of emulsified pomelo (Citrus grandis Osbeck) peel oil and water-soluble chitosan on Staphylococcus aureus and Escherichia coli. Molecules 23: 840, 2018.

30. Koleva II, van Beek TA, Linssen JP, de Groot A and Evstatieva LN: Screening of plant extracts for antioxidant activity: A comparative study on three testing methods. Phytochem Anal 13: 8-17, 2002.

31. Nguyen Thi PA, Chen MH, Li N, Zhuo XJ and Xie L: PD98059 protects brain against cells death resulting from ROS/ERK activation in a cardiac arrest rat model. Oxid Med Cell Longev 2016: $3723762,2016$.

32. Liu D, Wang H, Zhang Y and Zhang Z: Protective effects of chlorogenic acid on cerebral ischemia/reperfusion injury rats by regulating oxidative stress-related Nrf2 pathway. Drug Des Devel Ther 14: 51-60, 2020.

33. Roberto D, Micucci P, Sebastian T, Graciela F and Anesini C: Antioxidant activity of limonene on normal murine lymphocytes relation to $\mathrm{H}_{2} \mathrm{O}_{2}$ modulation and cell proliferation. Basic Clin Pharmacol Toxicol 106: 38-44, 2010.

34. Liao S, Wu J, Liu R, Wang S, Luo J, Yang Y, Qin Y, Li T, Zheng X, Song J, et al: A novel compound DBZ ameliorates neuroinflammation in LPS-stimulated microglia and ischemic stroke rats: Role of Akt(Ser473)/GSK3beta(Ser9)-mediated Nrf2 activation. Redox Biol 36: 101644, 2020.

35. Zhao J, Piao X, Wu Y, Liang S, Han F, Liang Q, Shao S and Zhao D: Cepharanthine attenuates cerebral ischemia/reperfusion injury by reducing NLRP3 inflammasome-induced inflammation and oxidative stress via inhibiting 12/15-LOX signaling. Biomed Pharmacother 127: 110151, 2020.

36. Swanson KV, Deng M and Ting JP: The NLRP3 inflammasome: Molecular activation and regulation to therapeutics. Nat Rev Immunol 19: 477-489, 2019.
37. Zhou R, Yazdi AS, Menu P and Tschopp J: A role for mitochondria in NLRP3 inflammasome activation. Nature 469: 221-225, 2011.

38. Dostert C, Pétrilli V, Van Bruggen R, Steele C, Mossman BT and Tschopp J: Innate immune activation through Nalp3 inflammasome sensing of asbestos and silica. Science 320: 674-677, 2008.

39. Groslambert M and Py BF: Spotlight on the NLRP3 inflammasome pathway. J Inflamm Res 11: 359-374, 2018.

40. Nakahira K, Haspel JA, Rathinam VA, Lee SJ, Dolinay T, Lam HC, Englert JA, Rabinovitch M, Cernadas M, Kim HP, et al: Autophagy proteins regulate innate immune responses by inhibiting the release of mitochondrial DNA mediated by the NALP3 inflammasome. Nat Immunol 12: 222-230, 2011.

41. Shimada K, Crother TR, Karlin J, Dagvadorj J, Chiba N, Chen S, Ramanujan VK, Wolf AJ, Vergnes L, Ojcius DM, et al: Oxidized mitochondrial DNA activates the NLRP3 inflammasome during apoptosis. Immunity 36: 401-414, 2012.

42. Parada E, Casas AI, Palomino-Antolin A, Gómez-Rangel V, Rubio-Navarro A, Farré-Alins V, Narros-Fernandez P, Guerrero-Hue M, Moreno JA, Rosa JM, et al: Early toll-like receptor 4 blockade reduces ROS and inflammation triggered by microglial pro-inflammatory phenotype in rodent and human brain ischaemia models. Br J Pharmacol 176: 2764-2779, 2019.

43. Meng H, Zhao H, Cao X, Hao J, Zhang H, Liu Y, Zhu MS, Fan L, Weng L, Qian L, et al: Double-negative T cells remarkably promote neuroinflammation after ischemic stroke. Proc Natl Acad Sci USA 116: 5558-5563, 2019.

44. Chen L, Kong L, Wei X, Wang Y, Wang B, Zhang X, Sun J and Liu $\mathrm{H}: \beta$-arrestin 2 negatively regulates NOD2 signalling pathway through association with TRAF6 in microglia after cerebral ischaemia/reperfusion injury. J Cell Mol Med 23: 3325-3335, 2019.

45. Wang Y, Li SY, Shen S and Wang J: Protecting neurons from cerebral ischemia/reperfusion injury via nanoparticle-mediated delivery of an siRNA to inhibit microglial neurotoxicity. Biomaterials 161: 95-105, 2018.

46. Bauernfeind F, Bartok E, Rieger A, Franchi L, Núñez G and Hornung V: Cutting edge: Reactive oxygen species inhibitors block priming, but not activation, of the NLRP3 inflammasome. J Immunol 187: 613-617, 2011.

47. Mandola AB, Sharfe N, Nagdi Z, Dadi H, Vong L, Merico D, Ngan B, Reid B and Roifman CM: Combined immunodeficiency caused by a novel homozygous NFKB1 mutation. J Allergy Clin Immunol: Sep 25, 2020 (Epub ahead of print). doi: 10.1016/j. jaci.2020.08.040.

48. Yi H, Peng R, Zhang LY, Sun Y, Peng HM, Liu HD, Yu LJ, Li AL, Zhang YJ, Jiang WH and Zhang Z: LincRNA-Gm4419 knockdown ameliorates NF- $\kappa \mathrm{B} / \mathrm{NLRP} 3$ inflammasome-mediated inflammation in diabetic nephropathy. Cell Death Dis 8: e2583, 2017.

49. Chen J, Yang C, Xu X, Yang Y and Xu B: The effect of focal cerebral ischemia-reperfusion injury on TLR4 and NF- $\kappa \mathrm{B}$ signaling pathway. Exp Ther Med 15: 897-903, 2018.

This work is licensed under a Creative Commons Attribution-NonCommercial-NoDerivatives 4.0 International (CC BY-NC-ND 4.0) License. 